This is the pre-peer reviewed version of the following article: Naqui, J, et al. "Coplanar waveguides loaded with symmetric and asymmetric pairs of slotted stepped impedance resonators: modeling, applications, and comparison to SIR-loaded CPWS" in Microwave and optical technology letters, vol. 58, issue 11 (Nov. 2016), p. 2741-2745, which has been published in final form at DOI 10.1002/ mop.30134. This article may be used for non-commercial purposes in accordance with Wiley terms and conditions for self-archiving.

\title{
Coplanar Waveguides (CPWs) Loaded with Symmetric and Asymmetric Pairs of Slotted Stepped Impedance Resonators (S-SIRs): Modeling, Applications and Comparison to SIR-loaded CPWs
}

\author{
Jordi Naqui, Lijuan Su, Javier Mata, and Ferran Martín \\ CIMITEC, Departament d'Enginyeria Electrònica \\ Universitat Autònoma de Barcelona, 08193 Bellaterra (Barcelona), Spain \\ Corresponding author: Ferran.Martin@uab.es \\ Fax: 34935812600
}

\begin{abstract}
This paper is focused on the analysis and modeling of coplanar waveguide (CPW) transmission lines loaded with pairs of slotted stepped impedance resonators (S-SIRs), etched in the ground planes. These structures may be described by a pair of parallel-connected transmission lines, each one loaded with a series-connected parallel resonator, and with mutual capacitive coupling between such resonators. If the structures are symmetric, the transmission coefficient exhibits a single transmission zero, related to the fundamental resonance of the SIRs, though perturbed by the effects of mutual coupling. However, if symmetry is disrupted, two notches appear, separated a distance that depends on the level of asymmetry, coupling, and CPW length. Therefore, these structures may be useful for the implementation of differential sensors and comparators. It is shown that if the S-SIR-loaded CPWs are electrically small, the structures can be modeled by a lumped element equivalent circuit. Using this model, the resonance frequencies are obtained analytically. The proposed lumped element model is validated through electromagnetic simulation and experiment, and a prototype comparator loaded with dielectric samples is fabricated and measured. Finally, the circuit model of the structure is compared to that of SIR-loaded CPW transmission lines. Despite the fact that the lumped element equivalent circuits of these structures are completely different, the equations providing the transmission zeros are formally identical.
\end{abstract}

KEYWORDS: Coplanar waveguide (CPW), stepped impedance resonators (SIRs), slot resonators, lumped element equivalent circuits. 


\section{Introduction}

Stepped impedance resonators (SIRs) are electrically small resonators useful for the implementation of planar filters in microstrip and coplanar waveguide (CPW) technology [1]. Although SIRs are usually implemented as metallic resonators, the complementary counterparts (slotted SIRs -S-SIRs) are also of interest for microwave circuit design. In particular, dumbbellshaped slotted SIRs symmetrically etched in the ground plane of a microstrip line are useful to create notches in the frequency response, and have been modeled as parallel resonant tanks series-connected to the line [2].

In this paper, we investigate the behavior of CPW transmission lines loaded with pairs of coupled S-SIRs for the implementation of differential microwave sensors and comparators. Such CPW structures can be an alternative to state-of-the-art microwave sensors and comparators for dielectric characterization of materials (organic tissue and biological sample analysis, defects or abnormalities in materials, etc.) [3], [4]. The main advantage of the proposed CPW structures is the major robustness against variable environmental conditions provided by a differential measurement.

Recently, a microstrip structure loaded with pairs of coupled metallic SIRs was investigated as a potential candidate for differential sensing [5], and a preliminary prototype sensor operative at the V-band was presented. As compared to the structures therein, the S-SIR-loaded CPW structures presented in this paper may take advantage of a superior sensitivity, as well as a simpler fabrication complexity due to the uni-planar metallic configuration of the CPW structure. The sensing principle is based on the relative variation of the edge capacitance of one of the slotted SIRs with regard to the other, experienced when a pair of samples under test (SUT) is located on top of them. It is expected that the effects of the SUT are more pronounced when they are placed on top of edge capacitances than on top of broad-side capacitances (as in SIR-loaded microstrip lines), and this explains the sensitivity enhancement expected for the structures studied in this paper. The sensing principle is thus based on symmetry disruption, causing the presence of two notches in the transmission coefficient separated a distance that depends (although not exclusively) on the level of asymmetry. A frequency-splitting sensing principle was proposed for the design of spatial sensors based on microstrip lines loaded with split ring resonators in [6].

The analysis and modeling of these CPW structures loaded with symmetric and asymmetric SSIR pairs is necessary for design purposes. This is the actual challenge of this work, where electric coupling between the two S-SIRs is included for an accurate modeling. The proposed lumped element equivalent circuit model is validated through parameter extraction and comparison of the circuit response (for both symmetric and asymmetric configurations) to electromagnetic simulations and measurements. The viability of the proposed structure as comparator is then demonstrated. Finally, these S-SIR-loaded CPWs are compared to CPWs loaded with SIRs (indeed 5-section SIRs - 5S-SIRs) etched in the back substrate side [7], where it will be shown that, subjected to certain element mapping, the equations giving the pair of transmission zero frequencies (asymmetric case) in both structures (inferred from the corresponding models) are identical. An explanation of this fact is also given at the end of the paper. 


\section{Topology and model of the S-SIR-loaded CPW}

Fig. 1 (a) shows the typical topology of a CPW loaded with a pair of S-SIRs. Although the topology shown in Fig. 1(a) is symmetric with regard to the line axis, the analysis will be carried out by considering the general case of arbitrary S-SIR dimensions. The modeling of CPWs loaded with slotted patterns (not necessarily S-SIRs), has been performed in several works [8]-[10]. However, in these publications the analysis corresponds to symmetric structures. Exceptionally, asymmetric structures providing more than one notch in the transmission coefficient are reported in [9]. Nevertheless, the reported model in [9] does not account for the effects of asymmetry in frequency splitting, and coupling between resonant elements is not modeled. In [11], a circuit model for a CPW-slotline cross was presented. The model accounts for the effects of asymmetry in the slotlines (generating the slot mode in the CPW structure), but this model is based on the decomposition of the two fundamental CPW modes (even and odd) into different ports, resulting in a complex 6-port circuit model, accurate but complex for design purposes.

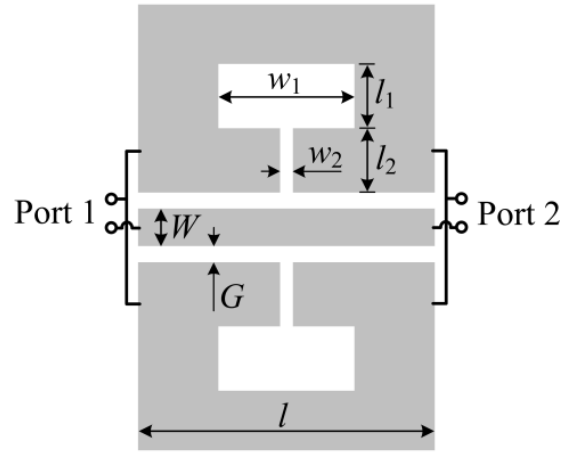

(a)

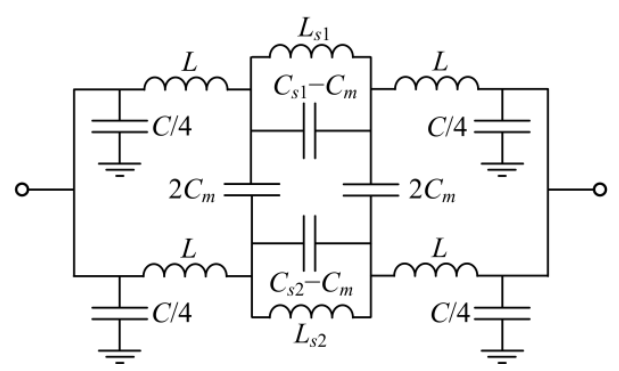

(c)

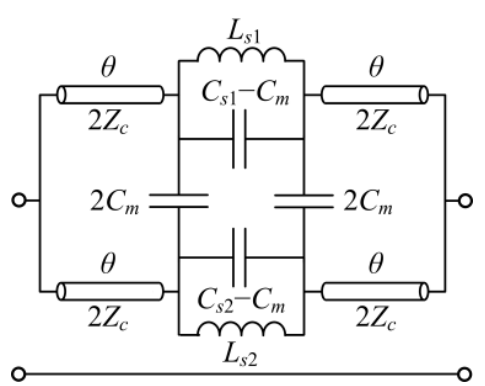

(b)

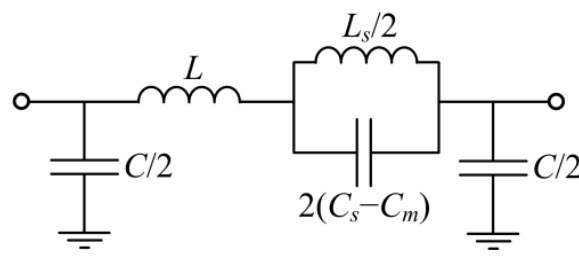

(d)

Fig. 1. (a) CPW loaded with a pair of coupled slotted SIRs; (b) hybrid lumped-distributed model; (c) lumped model; and (d) simplified lumped model for a symmetric pair of resonators.

The proposed model for the structure of Fig. 1(a) is depicted in Fig. 1(b). It is assumed that the structure can be described as a pair of split transmission lines (the central strip being a common conductor), each one loaded with a series-connected parallel resonant tank $\left(L_{s i} C_{s i}\right.$ with $\left.i=1,2\right)$. The electrical length and characteristic impedance of the transmission line sections are $\theta$ and $2 Z_{c}$ (where $Z_{c}$ is the CPW characteristic impedance), respectively. It has been found that for reasonable widths of the CPW central strip, electric coupling between the resonators arises, and this effect is accounted for by including a mutual capacitance, $C_{m}$, in the model [12],[13]. Except for the symmetric case, the analytical solution for the transmission zero frequencies 
(resonance frequencies from now on) in the circuit of Fig. 1(b) is not straightforward. However, if the CPW transmission line is electrically short, we can describe it by its inductance, $L$, and capacitance, $C$, resulting in the model depicted in Fig. 1(c). It is apparent that a necessary condition to connect capacitively the resonators (by $C_{m}$ ) is a non-zero potential difference between the two lateral metallic planes. Accordingly, the slot mode must propagate through the structure, and this occurs only when the structure is asymmetric. By contrast, for a symmetric structure (i.e. $L_{s 1}=L_{s 2} \equiv L_{s}$ and $C_{s 1}=C_{s 2} \equiv C_{s}$ ), the circuit model can be simplified to that shown in Fig. 1(d). Indeed, this is the typical circuit model of S-SIR-loaded transmission lines reported in the literature (where inter-resonator coupling only shifts the resonance frequency according to the proposed circuit model). Note that losses are not considered in the models.

The analytical calculation of the resonance frequencies in the circuit of Fig. 1(c) is cumbersome, but such frequencies can be expressed in a relatively closed form. To this end, we have obtained the reactance of the series branch in the $\pi$-model of Fig 1 (c). By calculating such reactance and the corresponding poles, a bi-quadratic equation results,

where the coefficients are:

$$
A \omega^{4}+B \omega^{2}+D=0
$$

$$
\begin{aligned}
& A=4 L L_{s 1} L_{s 2}\left(C_{s 1} C_{s 2}-C_{m}^{2}\right) \\
& B=-\left[L_{s 1} L_{s 2}\left(C_{s 1}+C_{s 2}\right)-2 L_{s 1} L_{s 2} C_{m}+4 L\left(C_{s 1} L_{s 1}+C_{s 2} L_{s 2}\right)\right] \\
& D=L_{s 1}+L_{s 2}+4 L
\end{aligned}
$$

and the solutions are of the form

$$
\omega_{ \pm}^{2}=\frac{A^{\prime} \pm \sqrt{B^{\prime}}}{D^{\prime}}
$$

with

$$
\begin{aligned}
& A^{\prime}=L_{s 1} L_{s 2}\left(C_{s 1}+C_{s 2}-2 C_{m}\right)+4 L\left(L_{s 1} C_{s 1}+L_{s 2} C_{s 2}\right) \\
& B^{\prime}=L_{s 1}^{2} L_{s 2}^{2}\left(C_{s 1}+C_{s 2}-2 C_{m}\right)^{2}+16 L^{2}\left(L_{s 1} C_{s 1}-L_{s 2} C_{s 2}\right)^{2}+8 L L_{s 1} L_{s 2} \times \\
& \left\{L_{s 1} C_{s 1}\left(C_{s 1}-C_{s 2}-2 C_{m}\right)+L_{s 2} C_{s 2}\left(C_{s 2}-C_{s 1}-2 C_{m}\right)+2 C_{m}^{2}\left(L_{s 1}+L_{s 2}+4 L\right)\right\} \\
& D^{\prime}=8 L L_{s 1} L_{s 2}\left(C_{s 1} C_{s 2}-C_{m}^{2}\right)
\end{aligned}
$$

If the structure is symmetric (i.e., $L_{s 1}=L_{s 2} \equiv L_{s}$ and $C_{s 1}=C_{s 2} \equiv C_{s}$ ), the mathematical solutions are:

$$
\begin{aligned}
& \omega_{+}=\frac{1}{\sqrt{\left(C_{s}+C_{m}\right) \frac{2 L L_{s}}{2 L+L_{s}}}} \\
& \omega_{-}=\frac{1}{\sqrt{L_{s}\left(C_{s}-C_{m}\right)}}=\frac{\omega_{0}}{\sqrt{1-k_{e}}}
\end{aligned}
$$

where $\omega_{0}=\left(L_{s} C_{s}\right)^{-1}$ and $k_{e}=C_{m} / C_{s}$ is the electric coupling coefficient. However, $\omega_{+}$is not actually a solution since it nulls the numerator of the reactance. This can also be derived by inspection of the circuit in Fig. 1(d). 


\section{Validation of the model}

The models of Fig. 1 have been validated by considering three CPW structures loaded with different pairs of S-SIRs (see Fig. 2): a symmetric pair, and two asymmetric pairs where the width $w_{2}$ of one of the resonators is modified (this width changes both $C_{s}$ and $L_{s}$ of the corresponding resonator). First, the lumped element values of the circuit in Fig. 1(d) have been obtained assuming symmetric pairs with $l=7 \mathrm{~mm}$. Thereby, for each $w_{2}$, the inductance, $L_{s}$, and the capacitance in the presence of coupling, $C_{\text {seff }}=C_{s}-C_{m}$, is obtained for each resonator. The parameters have been extracted from electromagnetic simulation (conductor and substrate losses are not considered) using the method reported in [14], [15]. Next, for the asymmetric structures, modeled by the circuit in Fig. 1(c), we have determined $C_{m}$ by curve fitting. The extracted parameters are indicated in the caption of Fig. 3. Finally, since the fabricated CPW structures are not electrically small, we have included four transmission line sections like those represented in Fig. 1(b). It should be noted that the resonance frequencies depend on the CPW length, $l$. All the frequency responses are plotted in Fig. 3, revealing that there is good agreement between simulations and measurements.

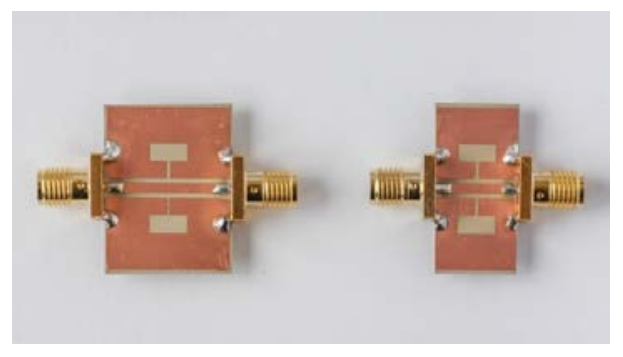

(a)

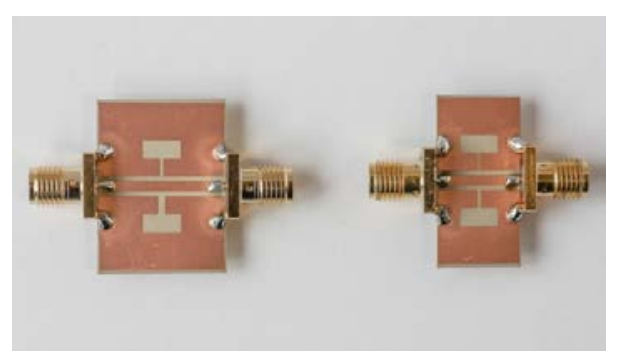

(b)

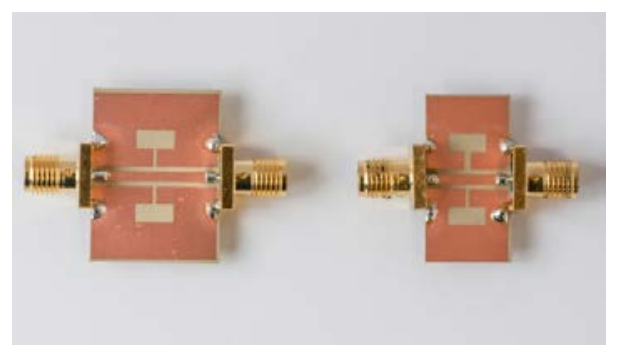

(c)

Fig. 2. Photograph of the fabricated $50-\Omega$ CPWs loaded with pairs of S-SIRs. The substrate is Rogers RO3010 with thickness $\mathrm{h}=1.27 \mathrm{~mm}$ and dielectric constant $\varepsilon \mathrm{r}=11.2$. Dimensions are: $\mathrm{W}=1.5 \mathrm{~mm}, \mathrm{G}=$ $0.65 \mathrm{~mm}, \mathrm{l}=12 \mathrm{~mm}, \mathrm{l} 1=\mathrm{l} 2=2.6 \mathrm{~mm}, \mathrm{w} 1=5.5 \mathrm{~mm}$, and for the lower fixed resonator $\mathrm{w} 2=0.5 \mathrm{~mm}$. For the upper resonator, w2 is (a) $0.5 \mathrm{~mm}$, (b) $0.25 \mathrm{~mm}$, and (c) $1 \mathrm{~mm}$. 
A potential application of these structures is related to differential sensors and comparators based on frequency-splitting, since two split resonance frequencies appear when symmetry is broken. Particularly, Fig. 4 shows the response of the symmetric structure of Fig. 2(a) when it is symmetrically loaded with a pair of dielectric slabs of dielectric constant 3.38, and when it is asymmetrically loaded with a single slab (see inset). In the latter case, the asymmetry is clearly manifested by the presence of two notches. Note that for the asymmetric case, the response overall shifts to the right since subtracting one slab is equivalent to reduce one of the S-SIR capacitances.

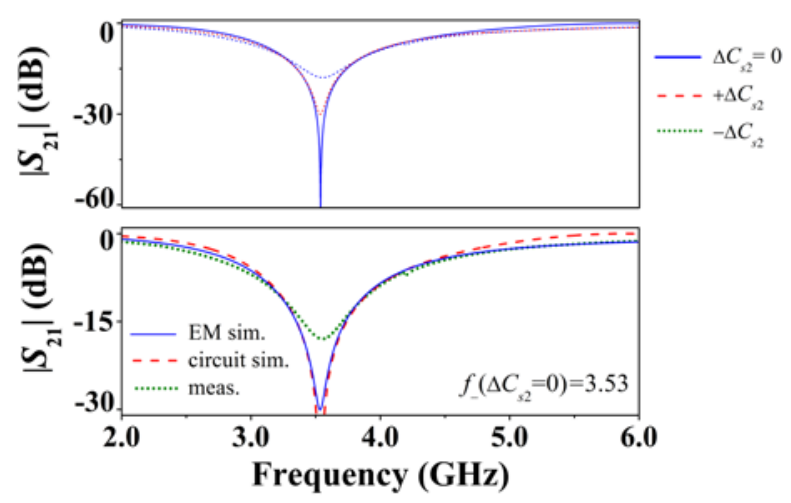

(a)

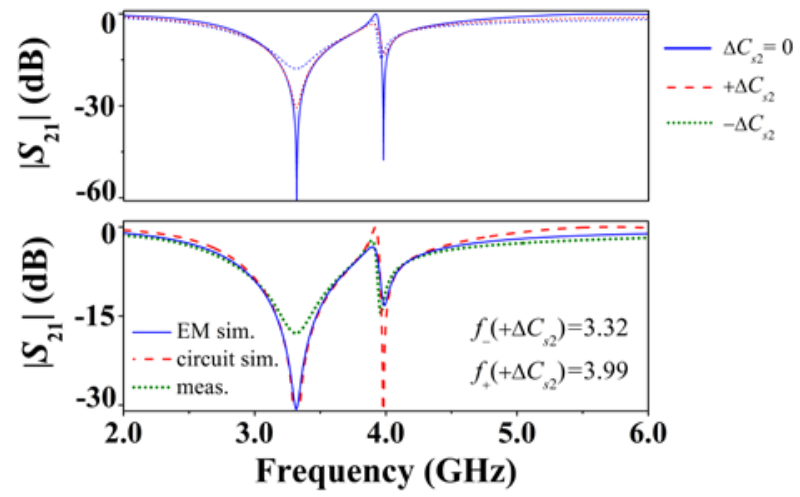

(b)

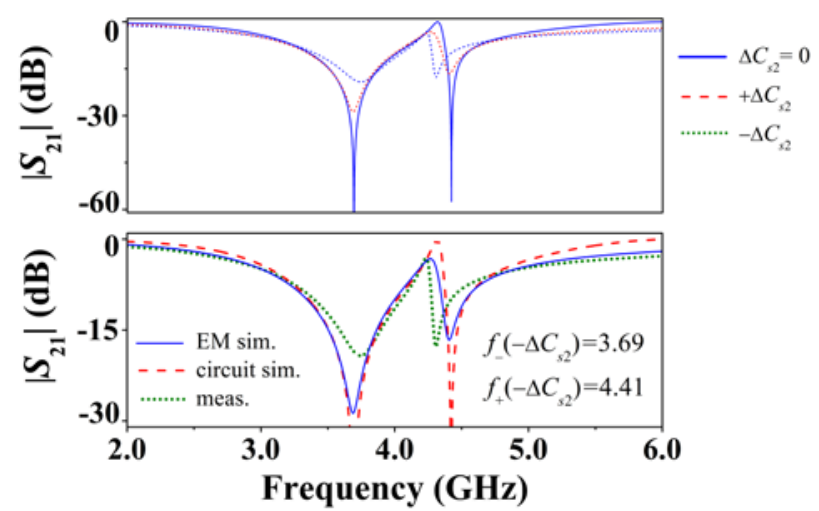

(c)

Fig. 3. Transmission coefficient magnitude for the structures in Fig. 2. The lumped parameters are: $\mathrm{L}=$ $2.36 \mathrm{nH}, \mathrm{C}=1.28 \mathrm{pF}$; for $\mathrm{w} 2=0.25 \mathrm{~mm}, \mathrm{Ls}=3.77 \mathrm{nH}$, Cseff $=0.66 \mathrm{pF}$; for $\mathrm{w} 2=0.5 \mathrm{~mm}$, Ls $=3.63 \mathrm{nH}$ 
and Cseff $=0.56 \mathrm{pF}$; for $\mathrm{w} 2=1 \mathrm{~mm}$, Ls $=3.39 \mathrm{nH}$ and Cseff $=0.46 \mathrm{pF} ; \mathrm{Cm}(+\Delta \mathrm{Cs} 2)=-0.09 \mathrm{pF}$ and $\mathrm{Cm}(-\Delta \mathrm{Cs} 2)=-0.08 \mathrm{pF}(\mathrm{Cseff}=\mathrm{Cs}-\mathrm{Cm}$ where $\mathrm{Cs}$ is the capacitance for a symmetric case $)$. The indicated resonance frequencies correspond to simulation.

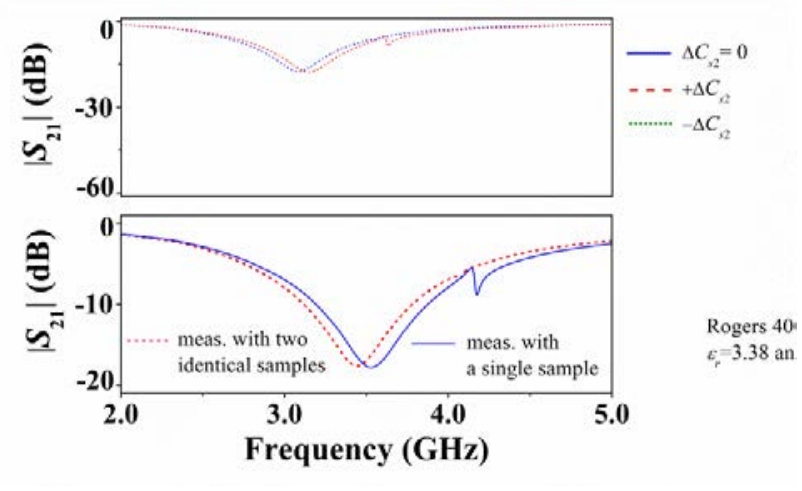

(a)

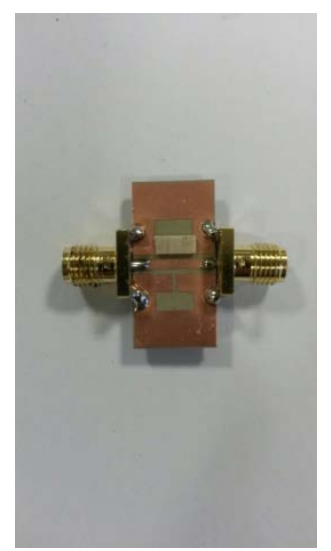

(b)

Fig. 4. Symmetric and asymmetric dielectric loading onto the structure of Fig. 2(a) by means of a pair and a single dielectric sample $(\varepsilon \mathrm{r}=3.38$ and $\mathrm{h}=0.8128 \mathrm{~mm})$, respectively.

\section{Comparison to SIR-loaded CPWs}

In [7], CPW transmission lines loaded with 5-section SIRs etched in the back substrate side were studied, and a circuit model for these lines was proposed. It is interesting to highlight that the bi-quadratic equation providing the transmission zeros in the SIR-loaded CPW is identical to equation (1) with the coefficients given by (2), provided the following mapping is satisfied: $C_{c}$ $\leftrightarrow 4 L ; C_{1,2} \leftrightarrow L_{s 1,2} ; L_{1,2} \leftrightarrow C_{s 1,2} ; M \leftrightarrow C_{m}$, where $C_{c}, C_{1,2}, L_{1,2}$ and $M$ are the reactive elements of the circuit model of the SIR-loaded CPW [7]. Thus, with this mapping, the pair of transmission zero frequencies of the two structures, given by (3) using (4) for S-SIR-loaded CPWs of the present paper, are also identical. Note, however, that the degenerate (and hence single) solutions for the symmetric cases occur at different frequencies (i.e., the upper frequency for the SIRloaded CPW and the lower frequency for the S-SIR-loaded CPW). This fact explains that for the 5-SIR-loaded CPW, when symmetry is disrupted, the two resulting notches are either located at both sides of the single notch corresponding to the symmetric case, or below it [7], whereas for the S-SIR-loaded CPW, when symmetry is disrupted, the two resulting notches are either located at both sides of the single notch corresponding to the symmetric case, or above it (see Fig. 3).

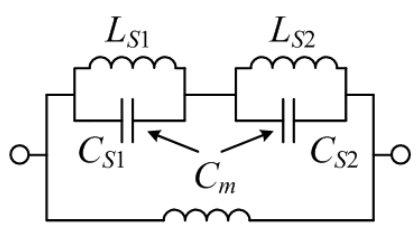

$4 L$

Fig. 5. Circuit dual of the resonant shunt branch of the equivalent circuit of the 5-SIR-loaded CPW of [7]. Note that the reactive elements have been designated according to the mapping.

Interestingly, despite the fact that the SIR- and S-SIR-loaded CPW transmission lines are significantly different, with the previous indicated mapping the expressions providing the resonance frequencies are identical for both structures. Neither these structures are dual, nor are the equivalent circuits circuit duals [16]. However, the poles of the dual circuit of the resonant 
shunt branch of the SIR-loaded CPW, depicted in Fig. 5, are identical to those of the circuit of Fig. 3(c), and this explains the formal coincidence of the two equations providing the transmission zero frequencies.

\section{Conclusion}

In this work, we have analyzed asymmetric CPW-based structures where the CPW central strip is kept unaltered whereas S-SIRs are etched on the lateral metallic planes (ground planes in conventional CPW implementations). A novel strategy for modeling symmetric/asymmetric configurations is reported, where the structures are viewed as two parallel-connected loaded transmission lines. A lumped element equivalent circuit of the structure has been proposed, and from this model the pair of transmission zeros have been analytically inferred. The model has been experimentally validated and the potential of the structure as differential sensor or comparator has been pointed out by means of a proof-of-concept device comparator. Finally, it has been demonstrated that the analytical expressions for the pair of transmission zeros in these S-SIR-loaded CPW structures are identical to those of the SIR-loaded CPWs, provided a simple mapping between elements is applied.

\section{Acknowledgements}

This work has been supported by MINECO (Spain) under project TEC2013-40600-R and FEDER Funds. Thanks are also given to AGAUR-Generalitat de Catalunya for partially funding this research activity through the project 2014SGR-157. Ferran Martín is in debt to ICREA for supporting his work. Lijuan Su thanks China Scholarship Council (CSC) for supporting her work in UAB through the grant No. 201306950011.

\section{References}

[1] M. Makimoto and S. Yamashita, Compact bandpass filters using stepped impedance resonators, Proc. IEEE, vol. 67, pp. 16-19, Jan. 1979.

[2] D. Ahn, J-S. Park, C-S. Kim, J. Kim, Y. Qian, and T. Itoh, A design of the low-pass filter using the novel microstrip defected ground structure, IEEE Trans. Microw. Theory Techn., vol. 49, pp. 86-93, Jan. 2001.

[3] M. Puentes, C. Weiß, M. Schüßler, and R. Jakoby, Sensor array based on split ring resonators for analysis of organic tissues, IEEE MTT-S International Microwave Symposium Digest, Baltimore, USA, June 2011.

[4] T. Chretiennot, D. Dubuc and K. Grenier, Optimized electromagnetic interaction microwave resonator/microfluidic channel for enhanced liquid bio-sensor, Proc. European Microwave Conference, Nuremberg, Germany, October 2013.

[5] J. Naqui, C. Damm, A. Wiens, R. Jakoby, L. Su, and F. Martín, Transmission lines loaded with pairs of magnetically coupled stepped impedance resonators (SIRs): modeling and application to microwave sensors, IEEE MTT-S Int. Microwave Symp., Jun. 2014, Tampa, FL (USA).

[6] A. K. Horestani, J. Naqui, Z. Shaterian, D. Abbott, C. Fumeaux, and F. Martín, Two-dimensional alignment and displacement sensor based on movable broadside-coupled split ring resonators, Sens. Act. A, vol. 210, pp. 18-24, Apr. 2014.

[7] L. Su, J. Naqui, J. Mata-Contreras, and F. Martín, Coplanar waveguides loaded with symmetric and asymmetric multisection stepped impedance resonators: modeling and potential applications, Microw. Opt. Technol. Lett., vol 58, pp. 722-726, Mar. 2016.

[8] J.-S. Lim, C.-S. Kim, Y-T. Lee, D. Ahn, and S. Nam, A Spiral-Shaped Defected Ground Structure for Coplanar Waveguide, IEEE Microw. Wirel. Compon. Lett., vol. 12, pp. 330-332, Sep. 2002. 
[9] A.M.E. Safwat, F. Podevin, P. Ferrari, and A. Vilcot, Tunable bandstop defected ground structure resonator using reconfigurable dumbbell-shaped coplanar waveguide, IEEE Trans. Microw. Theory Techn., vol. 54, pp. 3559-3564, Sep. 2006.

[10] A.M.E. Safwat, S. Tretyakov, and A.V. Räisänen, Defected ground and patch-loaded planar transmission lines, IET Microw. Antennas Propag., vol. 3, pp. 195-204, 2009.

[11] M. Ribó and L. Pradell, Circuit model for a coplanar-slotline cross, IEEE Microw. Guided Wave Lett., vol. 10, pp. 511-513, Dec. 2000.

[12] J.-S. Hong, and M. J. Lancaster, Microstrip Filters for RF/Microwave Applications, John Wiley \& Sons, 2001.

[13] Shuo Wang and F.C. Lee, Negative capacitance and its applications on parasitic cancellation for EMI Noise Suppression, IEEE PESC 2007, pp.2887-2891, Jun. 2007.

[14] J. Bonache, M. Gil, I. Gil, J. Garcia-García and F. Martín, On the electrical characteristics of complementary metamaterial resonators, IEEE Microw. Wireless Compon. Lett., vol. 16, pp. 543.545, Oct. 2006

[15] F. Aznar, M. Gil, J. Bonache, L. Jelinek, J. D. Baena, R. Marqués and F. Martín, Characterization of miniaturized metamaterial resonators coupled to planar transmission lines through parameter extraction, J. Appl. Phys., vol. 104, pp. 114501, Dec. 2008.

[16] W.J. Getsinger, Circuit duals on planar transmission media, IEEE MTT-S Int. Microwave Symp. Dig., May-June 1983, pp. 154-156, Boston, MA (USA). 